\title{
On radii of spheres determined by subsets of Euclidean space
}

\author{
Bochen Liu
}

\begin{abstract}
In this paper we consider the problem of how large the Hausdorff dimension of $E \subset \mathbb{R}^{d}$ needs to be in order to ensure that the radii set of $(d-1)$-dimensional spheres determined by $E$ has positive Lebesgue measure. We also study the question of how often can a neighborhood of a given radius repeat. We obtain two results. First, by applying a general mechanism developed in [4] for studying Falconer-type problems, we prove that a neighborhood of a given radius cannot repeat more often than the statistical bound if $\operatorname{dim}_{\mathcal{H}}(E)>d-1+\frac{1}{d} ;$ In $\mathbb{R}^{2}$, the dimensional threshold is sharp. Second, by proving an intersection theorem, we prove for a.e $a \in \mathbb{R}^{d}$, the radii set of $(d-1)$-spheres with center $a$ determined by $E$ must have positive Lebesgue measure if $\operatorname{dim}_{\mathcal{H}}(E)>d-1$, which is a sharp bound for this problem.
\end{abstract}

\section{Introduction}

The classical Falconer distance conjecture states that if a set $E \subset \mathbb{R}^{d}, d \geq 2$, has Hausdorff dimension greater than $\frac{d}{2}$, then the one-dimensional Lebesgue measure $\mathcal{L}^{1}(\Delta(E))$ of its distance set,

$$
\Delta(E):=\{|x-y| \in \mathbb{R}: x, y \in E\},
$$

is positive, where $|\cdot|$ denotes the Euclidean distance. Falconer gave an example based on the integer lattice showing that the exponent $\frac{d}{2}$ is best possible. The best results currently known, culminating almost three decades of efforts by Falconer [3], Mattila [11], Bourgain [1], and others, are due to Wolff [15] for $d=2$ and Erdoğan [2] for $d \geq 3$. They prove that $\mathcal{L}^{1}(\Delta(E))>0$ if

$$
\operatorname{dim}_{\mathcal{H}}(E)>\frac{d}{2}+\frac{1}{3} \text {. }
$$

It is natural to consider related problems, where distances determined by $E$ may be replaced by other geometric objects, such as triangles [5], volumes [6], angles [7], [8], and others. Generally, if

$$
\Phi:\left(\mathbb{R}^{d}\right)^{k+1} \rightarrow \mathbb{R}^{m}
$$

for some $1 \leq m \leq\left(\begin{array}{c}d+1 \\ 2\end{array}\right)$, we define a configuration set

$$
\Delta_{\Phi}(E)=\left\{\Phi\left(x^{1}, \ldots, x^{k+1}\right): x^{j} \in E\right\}
$$

2010 Mathematics Subject Classification. 28A75, 42B20.

Key words and phrases. Falconer-type problems, radii, intersection. 
and ask how large $\operatorname{dim}_{\mathcal{H}}(E)$ needs to be to ensure $\mathcal{L}^{m}\left(\Delta_{\Phi}(E)\right)>0$. For example, in the distance problem, $k=1, \Phi\left(x^{1}, x^{2}\right)=\left|x^{1}-x^{2}\right|$; in the volume problem, $k=d, \Phi\left(x^{1}, \ldots, x^{d+1}\right)=\mid \operatorname{det}\left(x^{d+1}-\right.$ $\left.x^{1}, \ldots, x^{d+1}-x^{d}\right) \mid$; in the angle problem, $k=1, \Phi\left(x^{1}, x^{2}\right)=\frac{x^{1} \cdot x^{2}}{\left|x^{1}\right|\left|x^{2}\right|}$.

In this paper, we consider the problem of the distribution of radii of spheres determined by $(d+1)$-tuples of points from $E$.

Definition 1.1. We say a sphere is determined by a $(d+1)$-tuple $\left(x^{1}, \ldots, x^{d+1}\right)$ if it is the unique sphere passing through all the points $x^{1}, \ldots, x^{d+1}$. We say a shpere is determined by a set $E$ if it is determined by a $(d+1)$-tuple $\left(x^{1}, \ldots, x^{d+1}\right) \in E \times \cdots \times E$.

Let $R\left(x^{1}, \ldots, x^{d+1}\right)$ be the radius of the unique $(d-1)$-dimensinal sphere determined by $\left(x^{1}, \ldots, x^{d+1}\right)$ and it equals 0 if such a sphere does not exist or it is not unique. We obtain two types of results. First, we estimate how often a neighborhood of a given radius occurs, in the sense defined below. Second, we find the optimal dimension such that the Lebesgue measure of the set of radii is positive. Our main geometric results are the following.

TheOREM 1.2. Let $E \subset \mathbb{R}^{d}$ be compact and $\nu$ be a Frostman measure on $E$. Then $\operatorname{dim}_{\mathcal{H}}(E)>$ $d-1+\frac{1}{d}$ implies

$$
(\nu \times \cdots \times \nu)\left\{\left(x^{1}, \ldots, x^{d+1}\right):\left|R\left(x^{1}, \ldots, x^{d+1}\right)-t\right|<\epsilon\right\} \lesssim \epsilon
$$

where the implicit constant is uniform in $t$ on any bounded set.

Moreover, $\operatorname{dim}_{\mathcal{H}}(E)>d-1+\frac{1}{d}$ implies

$$
\mathcal{L}(R(E \times \cdots \times E))>0 .
$$

When $d=k=2$, this result is sharp in the sense that (1.1) does not generally hold when $\operatorname{dim}_{\mathcal{H}}(E)<$ $\frac{3}{2}$.

In contrast to the incidence result, using Mattila's classical estimate on the dimension of intersections (see Theorem 5.1), one can prove that

$$
\operatorname{dim}_{\mathcal{H}}(E)>d-1 \Longrightarrow \mathcal{L}(R(E \times \cdots \times E))=\mathbb{R}^{+} .
$$

It's the optimal result because there is no unique $(d-1)$-dimensional sphere passing through $d+1$ points in a hyperplane. However, one can never get this bound by improving the incidence result above due to its sharpness.

We also prove an intersection result where rotations and translations are replaced by dilations and translations (see Theorem 5.4). From this intersection theorem we deduce the following.

TheOREm 1.3. Given $E \subset \mathbb{R}^{d}$ with $\operatorname{dim}_{\mathcal{H}}(E)>d-1$, then for a.e. $a \in \mathbb{R}^{d}$,

$$
\mathcal{L}\left(\left\{r>0: E \text { determines } S_{a, r}^{d-1}\right\}\right)>0,
$$

where $S_{a, r}^{d-1}$ is the $(d-1)$-dimensional sphere with center a and radius $r$.

The bound $d-1$ is sharp because a hyperplane can never determine a $(d-1)$-dimensional sphere of finite radius.

Notation. Throughout the paper,

$X \lesssim Y$ means that there exists $C>0$ such that $X \leq C Y$.

$S^{d-1}=\left\{x \in \mathbb{R}^{d}:|x|=1\right\}$. 
For $A \subset \mathbb{R}^{d}, A_{a, r}=\{r x+a: x \in A\}$. $q(x)$.

For a measure $\mu$ on $\mathbb{R}^{d}$ and a function $q(x)$ on $\mathbb{R}^{d}, q_{*} \mu$ is the measure on $q\left(\mathbb{R}^{d}\right)$ induced by

$\hat{\mu}(\xi)=\int e^{-2 \pi i x \cdot \xi} d x$ is the Fourier transform of the measure $\mu$.

Acknowledgement. The author wishes to thank Professor Alex Iosevich for suggesting this problem and comments that helped improve the manuscript.

\section{The Incidence Result}

In [4], Grafakos, Greenleaf, Iosevich, and Palsson develop the following general mechanism to solve Falconer-type problems.

We say $\Phi$ is translation invariant if it can be written as

$$
\Phi_{0}\left(x^{k+1}-x^{1}, \ldots, x^{k+1}-x^{k}\right) .
$$

Theorem 2.1 (Grafakos, Greenleaf, Iosevich, and Palsson, 2012). Suppose $\Phi$ is translation invariant and for some $\gamma>0$,

$$
\begin{aligned}
\left|\widehat{\mu_{t}}(-\xi, \xi, 0, \ldots, 0)\right| & \lesssim(1+|\xi|)^{-\gamma}, \\
\left|\widehat{\mu_{t}}(0, \xi, 0, \ldots, 0)\right| & \lesssim(1+|\xi|)^{-\gamma}, \\
\left|\widehat{\mu_{t}}(\xi, 0,0, \ldots, 0)\right| & \lesssim(1+|\xi|)^{-\gamma},
\end{aligned}
$$

where $\mu_{t}$ is the natural measure on $\left\{u: \Phi_{0}(u)=t\right\}$ and the implicit constant is uniform in $t$ on any bounded set. Then $\operatorname{dim}_{\mathcal{H}}(E)>d-\frac{\gamma}{k}$ implies

$$
(\nu \times \cdots \times \nu)\left\{\left(x^{1}, \ldots, x^{k+1}\right):\left|\Phi\left(x^{1}, \ldots, x^{k+1}\right)-t\right|<\epsilon\right\} \lesssim \epsilon^{m}
$$

where $\nu$ is a Frostman measure on $E$ and the implicit constant is uniform in $t$ in any bounded set. It follows that the $m$-dimensional Lebesgue measure $\mathcal{L}^{m}\left(\Delta_{\Phi}(E)\right)>0$.

\section{Proof of Theorem 1.2}

Since $R\left(x^{1}, \ldots, x^{d+1}\right)$ is translation invariant and it can be written as $R_{0}\left(x^{d+1}-x^{1}, \ldots, x^{d+1}-\right.$ $\left.x^{d}\right)$, by Theorem 2.1 it suffices to show all the three inequalities in (2.1) hold for the natural measure $\mu_{1}$ on $\left\{\left(u_{0}, \ldots, u_{d}\right) \in \mathbb{R}^{d}: R_{0}\left(u^{1}, \ldots, u^{d}\right)=1\right\}$. Observe that

a $(d+1)$-tuple $\left(x^{1}, \ldots, x^{d+1}\right)$ determines a sphere with radius 1

$\Leftrightarrow\left(0, x^{d+1}-x^{1}, \ldots, x^{d+1}-x^{d}\right)$ determines a sphere with radius 1 passing through the origin

$\Leftrightarrow x^{d+1}-x^{i} \in a+S^{d-1}$ for some $a \in S^{d-1}$ and $0 \neq x^{d+1}-x^{i} \neq x^{d+1}-x^{j}, \forall i \neq j$.

Hence

$$
\left\{\left(u^{1}, \ldots, u^{d}\right): R_{0}\left(u^{1}, \ldots, u^{d}\right)=1\right\}=\left\{\left(\sigma_{0}+\sigma_{1}, \sigma_{0}+\sigma_{2}, \ldots, \sigma_{0}+\sigma_{d}\right): \sigma_{i} \in S^{d-1}\right\}-N,
$$

where $N$ is a set of measure 0 . 
Let $\nu$ be the natural probability measure on $S^{d-1}$ and $\psi$ be some smooth cut-off function which may vary from line to line. By changing variables as (3.1),

$$
\begin{aligned}
\left|\widehat{\mu_{1}}(\xi,-\xi, 0, \ldots, 0)\right| & =\left|\int_{\left\{u: R_{0}(u)=1\right\}} e^{-2 \pi i u \cdot(\xi,-\xi, 0, \ldots, 0)} d u\right| \\
& =\left|\int \ldots \int e^{-2 \pi i \xi \cdot\left(\sigma_{1}-\sigma_{2}\right)} \psi d \nu\left(\sigma_{0}\right) \ldots d \nu\left(\sigma_{d}\right)\right| \\
& \lesssim\left|\iint e^{-2 \pi i \xi \cdot\left(\sigma_{1}-\sigma_{2}\right)} \psi d \nu\left(\sigma_{1}\right) d \nu\left(\sigma_{2}\right)\right| \\
& =\left|\int_{S^{d-1}} e^{-2 \pi \xi \cdot \sigma_{1}}\left(\int_{S^{d-1}} e^{2 \pi i \xi \cdot \sigma_{2}} \psi d \nu\left(\sigma_{2}\right)\right) d \nu\left(\sigma_{1}\right)\right| \\
& =\left|\int_{S^{d-1}} e^{-2 \pi \xi \cdot \sigma_{1}} a\left(\xi, \sigma_{1}\right) d \nu\left(\sigma_{1}\right)\right|
\end{aligned}
$$

By stationary phase $[13],\left|a\left(\xi, \sigma_{1}\right)\right| \lesssim(1+|\xi|)^{-\frac{d-1}{2}}$ and

$$
\left|\int_{S^{d-1}} e^{-2 \pi \xi \cdot \sigma_{1}}\left((1+|\xi|)^{\frac{d-1}{2}} a\left(\xi, \sigma_{1}\right)\right) d \nu\left(\sigma_{1}\right)\right| \lesssim(1+|\xi|)^{-\frac{d-1}{2}}
$$

Hence

$$
\left|\widehat{\mu_{1}}(\xi,-\xi, 0, \ldots, 0)\right| \lesssim(1+|\xi|)^{-(d-1)}
$$

For $\widehat{\mu_{1}}(\xi, 0, \ldots, 0)$ and $\widehat{\mu_{1}}(0, \xi, 0, \ldots, 0)$, after changing variables as above we have

$$
\begin{aligned}
\widehat{\mu_{1}}(\xi, 0, \ldots, 0) & =\int \ldots \int e^{-2 \pi i \xi \cdot\left(\sigma_{0}+\sigma_{1}\right)} \psi d \nu\left(\sigma_{0}\right) \ldots d \nu\left(\sigma_{d}\right), \\
\widehat{\mu_{1}}(0, \xi, 0, \ldots, 0) & =\int \ldots \int e^{-2 \pi i \xi \cdot\left(\sigma_{0}+\sigma_{2}\right)} \psi d \nu\left(\sigma_{0}\right) \ldots d \nu\left(\sigma_{d}\right) .
\end{aligned}
$$

By a similar argument,

$$
\left|\widehat{\mu_{1}}(\xi, 0, \ldots, 0)\right|,\left|\widehat{\mu_{1}}(0, \xi, 0, \ldots, 0)\right| \lesssim(1+|\xi|)^{-(d-1)},
$$

which completes the proof of Theorem 1.2.

\section{Sharpness of Theorem 1.2 in $\mathbb{R}^{2}$}

When $d=2$, Theorem 1.2 says $\operatorname{dim}_{\mathcal{H}}(E)>\frac{3}{2}$ implies $(2.2)$, more precisely

$$
(\nu \times \nu \times \nu)\left\{\left(x^{1}, x^{2}, x^{3}\right):\left|R\left(x^{1}, x^{2}, x^{3}\right)-t\right|<\epsilon\right\} \lesssim \epsilon
$$

where $\nu$ is a Frostman measure on a compact set $E \subset \mathbb{R}^{2}$ and the implicit constant is uniform in $t$ on any bounded set. Now we will show $\frac{3}{2}$ is sharp for (4.1) with a counterexample motivated by Mattila [11].

Without loss of generality, fix $t=100$. Let $C_{\alpha} \subset[0,1]$ denote the Cantor set of dimension $\alpha$ and $\nu$ denote the natural probability measure on $C_{\alpha}$. Let

$$
E=\left(\cup_{k=-200}^{200}\left(C_{\alpha}+k\right)\right) \times[-200,200]
$$

and extend $\nu$ to $E$ in the natural way. 


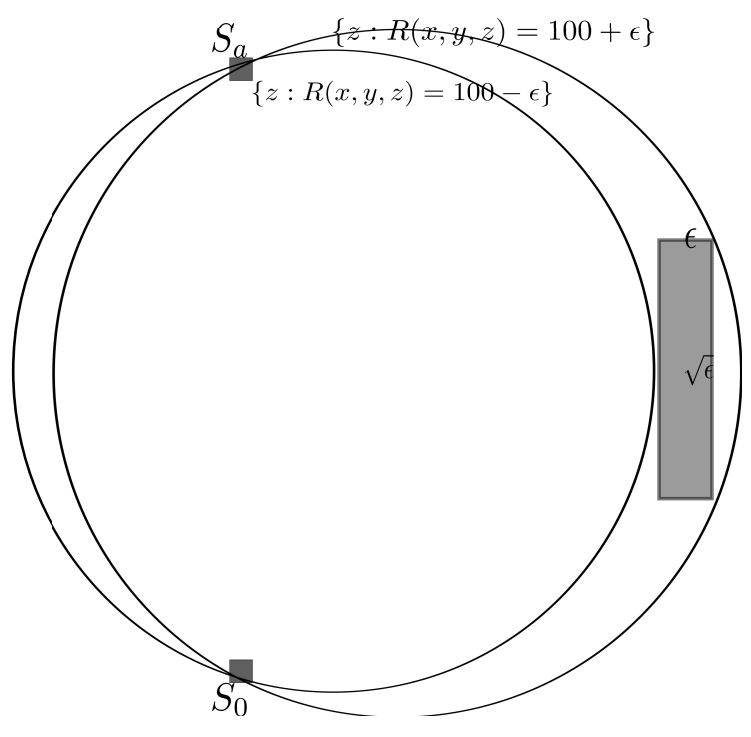

Let

$$
S_{a}=\left\{(x, y) \in \mathbb{R}^{2}: 0 \leq x \leq 1, a \leq y \leq a+1\right\} .
$$

When $a \gg 1$, for all $x \in S_{0}, y \in S_{a}$, the angle between the $y$-axis and the vector $y-x$ is very small. Therefore we can pick a $\sqrt{\epsilon} \times \epsilon$ rectangle in

$$
\left\{z \in \mathbb{R}^{2}:|R(x, y, z)-100|<\epsilon\right\}
$$

such that its $\sqrt{\epsilon}$-edges are parallel to the $y$-axis. (see the figure above).

If $a$ changes continuously, the $\sqrt{\epsilon} \times \epsilon$ rectangle translates continuously without any rotation. Then there exsit an $a_{0} \in(a-2, a)$ such that one of its $\sqrt{\epsilon}$-edges lies on a line $x=n$ for some $n \in \mathbb{Z}$. By the construction of $E$, for all $x \in S_{0}, y \in S_{a_{0}}$,

$$
\nu\left\{z \in \mathbb{R}^{2}:|R(x, y, z)-100|<\epsilon\right\} \gtrsim \epsilon^{\frac{1}{2}} \cdot \epsilon^{\alpha}=\epsilon^{\frac{1}{2}+\alpha} .
$$

Hence

$$
\begin{aligned}
& \nu \times \nu \times \nu\{(x, y, z):|R(x, y, z)-100|<\epsilon\} \\
& \gtrsim \times \nu \times \nu\left\{(x, y, z): x \in S_{0}, y \in S_{a},|R(x, y, z)-100|<\epsilon\right\} \\
& \gtrsim \epsilon^{\frac{1}{2}+\alpha}
\end{aligned}
$$

which implies that when $\alpha<\frac{1}{2}$, (4.1) fails.

It follows that for $\operatorname{dim}_{\mathcal{H}}(E)<\frac{3}{2}$, (4.1) is not generally true, which proves the sharpness arguement of Theorem 1.2.

Remark 4.1. The sharpness of (4.1) doesn't mean $\frac{3}{2}$ is the best possible for radii problem, but for the method we use, it cannot be, generally, improved.

REMARK 4.2. The example above cannot show the sharpness in higher dimensions. For example, in $\mathbb{R}^{3}$, the rectangle we pick will become a $\sqrt{\epsilon} \times \sqrt{\epsilon} \times \epsilon$ rectangle. If we still construct a set like 
$C_{\alpha} \times C_{\beta} \times C_{\gamma}$, we will have a lower bound $\epsilon^{\frac{\alpha}{2}+\frac{\beta}{2}+\gamma}$ for (2.2). In nontrivial cases (i.e. $\operatorname{dim}_{\mathcal{H}}(E)>2$ ) it is always true that $\frac{\alpha}{2}+\frac{\beta}{2}+\gamma>\frac{1}{2}(\alpha+\beta+\gamma)>1$, which does not contradict the upper bound. In fact, in many relavant problems, a similar example can show the sharpness in $d=2$ but fail in $d \geq 3$ (see, e.g. [5], [11]).

\section{An Intersection Theorem}

Given $A, B \subset \mathbb{R}^{d}$, we consider the behavior of the intersection of $A \cap T_{\alpha}(B)$, where $T_{\alpha}$ is some family of transformations. Mattila $([\mathbf{1 1}],[\mathbf{1 0}])$ proves a general intersection theorem for orthogonal transformations.

Theorem 5.1 (Mattila, 1984). In $\mathbb{R}^{d}$, let $s, t>0, s+t>d$ and $t>\frac{d+1}{2}$. If $A, B \subset \mathbb{R}^{d}$ are Borel sets with $\mathcal{H}^{s}(A)>0, \mathcal{H}^{t}(B)>0$, then for $\theta_{d}$ almost all $g \in O(d)$,

$$
\mathcal{L}\left(\left\{a \in \mathbb{R}^{d}: \operatorname{dim}_{\mathcal{H}}(A \cap(g B+a) \geq s+t-d\}\right)>0,\right.
$$

where $\theta_{d}$ is the Haar measure on $O(d)$.

There are also intersection theorems on larger transformation groups, e.g. similarities ([9], $[10])$.

TheOREm 5.2 (Kahane,1986). Let $G$ be a closed subgroup of $G L(n, \mathbb{R})$ and let $\tau$ be a Haar measure. Let $E$ and $F$ be two $\sigma$-compact subsets of $\mathbb{R}^{d}$. Then for $\tau$-almost all $g \in G$ and $\epsilon>0$,

$$
\mathcal{L}\left(\left\{a \in \mathbb{R}^{d}: \operatorname{dim}_{\mathcal{H}}(E \cap(g F+a)) \geq \operatorname{dim}_{\mathcal{H}}(E)+\operatorname{dim}_{\mathcal{H}}(F)-d-\epsilon\right\}\right)>0 .
$$

From Theorem 5.1, we know when $d=2$, for every $r>0$, there exists some $a \in \mathbb{R}^{2}$ such that $\operatorname{dim}_{\mathcal{H}}\left(E \cap S_{a, r}^{d-1}\right) \geq \operatorname{dim}_{\mathcal{H}}(E)-1>0$. Hence (1.2) holds for $d=2$. In higher dimensions it is followed by the following lemma.

Lemma 5.3. Suppose $F \subset S^{d-1} \subset \mathbb{R}^{d}$ and $\operatorname{dim}_{\mathcal{H}}(F)>d-2$, then $F$ uniquely determines $S^{d-1}$, i.e. $S^{d-1}$ is the only sphere which contains all the points of $F$.

Proof. It is followed by induction. For $d=2$, it's trivial because $F$ has at least three points.

Suppose it holds in $\mathbb{R}^{k-1}$. For $F \subset S^{k-1} \subset \mathbb{R}^{k}$ with $\operatorname{dim}_{\mathcal{H}}(F)>k-2$, there exists a hyperplane $P$ such that $\operatorname{dim}_{\mathcal{H}}(P \cap F)>k-3$ (see, e.g. [12]. Then $P \cap F$ determines the lower dimensional sphere $P \cap S^{k-1}$. Hence $F$ determines $S^{k-1}$ because there is at least one point in $F \backslash(P \cap F)$.

Note all the intersection results above try to determine when the translation set has positive Lebesgue measure. Now let us consider when the dilation set has positive Lebesgue measure.

TheOREM 5.4. Suppose $E \subset \mathbb{R}^{d}$ is compact with $\operatorname{dim}_{\mathcal{H}}(E)=s>1$ and $\Gamma \subset \mathbb{R}^{d}$ is a smooth hypersurface with nonzero Gaussian curvature. Then

$$
\left.\left.\mathcal{L}\left(\left\{r \in \mathbb{R}: \operatorname{dim}_{\mathcal{H}}\left(E \cap \Gamma_{a, r}\right)\right\}\right) \geq s-1\right\}\right)>0
$$

holds for a.e. $a \in\left\{z \in \mathbb{R}^{d}: E \subset \bigcup_{r \in \mathbb{R}} \Gamma_{z, r}\right\}$.

In particularly, letting $\Gamma=S^{d-1}$, Theorem 1.3 follows from Theorem 5.4 and Lemma 5.3. 


\section{Proof of Theorem 5.4}

If $E \subset \bigcup_{r \in \mathbb{R}} \Gamma_{a, r}$ for some $a \in \mathbb{R}^{d}$, by the compactness of $E$, it suffices to consider the case that $\Gamma$ is bounded and on any line passing through $a$ there is at most one point of $\Gamma$.

Let $\sigma$ denote the surface measure on $\Gamma$. Let $\left\{p_{i}\right\}$ be a partition of unity on $\Gamma$ such that in the support of each $p_{i}$, $\Gamma$ has a local coordinate system $u^{i}=\left(u_{1}^{i}, \ldots, u_{d-1}^{i}\right)$. Thus we have a well-defined coordinate system for the cone $C_{i}=\left\{r x: x \in \operatorname{supp} p_{i}, r \in \mathbb{R}-\{0\}\right\}$. We can also extend $p_{i}$ to $C_{i}$ by setting $p_{i}(r x)=p(x), x \in \Gamma$. By changing variables $x=r x\left(u^{i}\right)$ in each $C_{i}$, we have

$$
\begin{aligned}
\int_{\bigcup_{r} \Gamma_{a, r}} f(x) d x & =\sum_{i} \int_{C_{i}} p_{i}(x-a) f(x) d x \\
& =\sum_{i} \iint p_{i}\left(r x\left(u^{i}\right)\right) f\left(r x\left(u^{i}\right)+a\right)|r|^{d-1} \phi_{i}\left(u^{i}\right) d u^{i} d r \\
& =\sum_{i} \int_{\mathbb{R}} \int_{\operatorname{supp} p_{i}} p_{i}(x) f(r x+a)|r|^{d-1} \tilde{\phi}_{i}(x) d \sigma(x) d r \\
& =\int_{\mathbb{R}} \int_{\Gamma} f(r x+a)|r|^{d-1} \psi(x) d \sigma(x) d r,
\end{aligned}
$$

where $\phi_{i}, \tilde{\phi}_{i}, \psi$ are smooth cut-off functions.

Since $\operatorname{dim}_{\mathcal{H}}(E)=s>1$, for every $\epsilon>0$, there exists a measure $\mu$ on $E$ such that the $(s-\epsilon)$ -

energy $I_{s-\epsilon}(\mu)<\infty$. Let $q(x)=r x+a$. Define measures $\sigma_{a, r}$ on $\Gamma_{a, r}$ and $\mu_{a, r}$ on $E \cap \Gamma_{a, r}$ by

$$
\begin{aligned}
\sigma_{a, r} & =q_{*}(\psi \sigma), \\
\mu_{a, r} & =\lim _{\delta \rightarrow 0} \mu * \rho_{\delta} d \sigma_{a, r},
\end{aligned}
$$

where $\rho_{\delta}(x)=\delta^{-d} \rho\left(\frac{x}{\delta}\right), \rho \in C_{0}^{\infty}$ and $\int \rho=1$.

Let

$$
g(r)= \begin{cases}|r|^{d-1} \Phi(r) & \text { if }-1<r<1 \\ \Phi(r) & \text { otherwise }\end{cases}
$$

where $\Phi \in L^{1}$ and $\Phi>0$ everywhere.

LEMma 6.1. Under notations above,

$$
\iint g(r) I_{s-1-\epsilon}\left(\mu_{a, r}\right) d r d a<\infty
$$




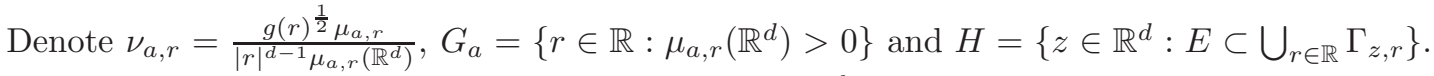
From Lemma $6.1, I_{s-1-\epsilon}\left(\mu_{a, r}\right)<\infty$ for a.e. $(r, a) \in \mathbb{R} \times \mathbb{R}^{d}$. Then by (6.1), (6.2), for $a \in H$,

$$
\begin{aligned}
1 & =\left(\mu\left(\mathbb{R}^{d}\right)\right)^{2} \\
& =\left(\lim _{\delta \rightarrow 0} \int_{\bigcup_{r} \Gamma_{a, r}} \mu * \rho_{\delta}(x) d x\right)^{2} \\
& =\left(\lim _{\delta \rightarrow 0} \iint \mu * \rho_{\delta}(r x+a)|r|^{d-1} \psi(x) d \sigma(x) d r\right)^{2} \\
& =\left(\lim _{\delta \rightarrow 0} \iint \mu * \rho_{\delta}(x)|r|^{d-1} d \sigma_{a, r}(x) d r\right)^{2} \\
& =\left(\int|r|^{d-1} \mu_{a, r}\left(\mathbb{R}^{d}\right) d r\right)^{2} \\
& =\left(\int_{G_{a}}|r|^{d-1} \mu_{a, r}\left(\mathbb{R}^{d}\right) I_{s-1-\epsilon}^{-\frac{1}{2}}\left(\mu_{a, r}\right) I_{s-1-\epsilon}^{\frac{1}{2}}\left(\mu_{a, r}\right) d r\right)^{2} \\
& =\left(\int_{G_{a}} I_{s-1-\epsilon}^{-\frac{1}{2}}\left(\nu_{a, r}\right) g(r)^{\frac{1}{2}} I_{s-1-\epsilon}^{-\frac{1}{2}}\left(\mu_{a, r}\right) d r\right)^{2} \\
& \leq \int_{G_{a}} I_{s-1-\epsilon}^{-1}\left(\nu_{a, r}\right) d r \int_{G_{a}} g(r) I_{s-1-\epsilon}\left(\mu_{a, r}\right) d r .
\end{aligned}
$$

Therefore,

$$
\begin{aligned}
\int_{H}\left(\int_{G_{a}} I_{s-1-\epsilon}^{-1}\left(\nu_{a, r}\right) d r\right)^{-1} d a & \leq \int_{H} \int_{G_{a}} g(r) I_{s-1-\epsilon}\left(\mu_{a, r}\right) d r d a \\
& \leq \iint g(r) I_{s-1-\epsilon}\left(\mu_{a, r}\right) d r d a \\
& <\infty .
\end{aligned}
$$

It follows that for a.e. $a \in \mathbb{R}^{d}, \int_{G_{a}} I_{s-1-\epsilon}^{-1}\left(\nu_{a, r}\right) d r>0$. Hence for a.e. $a \in \mathbb{R}^{d}, \mathcal{L}\left(G_{a}\right)>0$ and $\operatorname{dim}_{\mathcal{H}}\left(E \cap \Gamma_{a, r}\right) \geq s-1-\epsilon$ for a.e. $r \in G_{a}$. Since $G_{a}$ is independent of $\epsilon$, by choosing a sequence $\epsilon_{j} \rightarrow 0$, we complete the proof of Theorem 5.4. 


\section{Proof of Lemma 6.1}

From the the well-known equality $I_{\alpha}(\mu)=c_{\alpha, d} \int|\hat{\mu}(\xi)||\xi|^{-d+\alpha} d \xi$ (see, e.g. [12]) and Plancherel, we have

$$
\begin{aligned}
& \iint g(r) I_{s-1-\epsilon}\left(\mu_{a, r}\right) d r d a \\
= & c_{\alpha, d} \iiint g(r)\left|\hat{\mu} * \widehat{\sigma_{a, r}}(\xi)\right|^{2}|\xi|^{-d-1+s-\epsilon} d \xi d r d a \\
= & c_{\alpha, d} \iiint g(r)\left|\int \hat{\mu}(\eta) \widehat{\psi \sigma}(r(\xi-\eta)) e^{-2 \pi i a \cdot(\xi-\eta)} d \eta\right|^{2}|\xi|^{-d-1+s-\epsilon} d \xi d r d a \\
= & c_{\alpha, d} \iint\left(\int\left|\int \hat{\mu}(\eta) \widehat{\psi \sigma}(r(\xi-\eta)) e^{2 \pi i a \cdot \eta} d \eta\right|^{2} d a\right) g(r)|\xi|^{-d-1+s-\epsilon} d \xi d r \\
= & c_{\alpha, d} \iint\left(\int|\hat{\mu}(a) \widehat{\psi \sigma}(r(\xi-a))|^{2} d a\right) g(r)|\xi|^{-d-1+s-\epsilon} d \xi d r
\end{aligned}
$$

Since $\Gamma$ is smooth with nonzero Gaussian curvature everywhere, by stationary phase $[\mathbf{1 4}]$ and the construction of $g$,

$$
\int|\widehat{\psi \sigma}(r(\xi-a))|^{2} g(r) d r \lesssim|\xi-a|^{-(d-1)}
$$

Thus, to prove Lemma 6.1, it suffices to show

$$
\iint|\hat{\mu}(a)|^{2}|\xi-a|^{-(d-1)}|\xi|^{-d-1+s-\epsilon} d a d \xi<\infty
$$

For each $a \neq 0$, let $\xi=|a| \zeta$, then

$$
\int|\xi-a|^{-(d-1)}|\xi|^{-d-1+s-\epsilon} d \xi=|a|^{-d+s-\epsilon} \int\left|\zeta-\frac{a}{|a|}\right|^{-(d-1)}|\zeta|^{-d-1+s-\epsilon} d \zeta .
$$

When $\epsilon$ is small, $1<s-\epsilon<d$, then $\int\left|\zeta-\frac{a}{|a|}\right|^{-(d-1)}|\zeta|^{-d-1+s-\epsilon} d \zeta<\infty$ uniformly because $\frac{a}{|a|} \in S^{d-1}$ which is compact. Hence

$$
\begin{aligned}
\iint|\hat{\mu}(a)|^{2}|\xi-a|^{-(d-1)}|\xi|^{-d-1+s-\epsilon} d a d \xi & \lesssim \int|\hat{\mu}(a)|^{2}|a|^{-d+s-\epsilon} d a \\
& <\infty,
\end{aligned}
$$

which proves (7.2) and completes the proof of Lemma 6.1. 


\section{References}

[1] J. Bourgain, Hausdorff dimension and distance sets Israel. J. Math. 87 (1994), 193-201. 1

[2] B. Erdoğan, A bilinear Fourier extension theorem and applications to the distance set problem, Int. Math. Res. Not. (2005), no. 23, 1411-1425. 1

[3] K. J. Falconer On the Hausdorff dimensions of distance sets, Mathematika 32 (1986), 206-212. 1

[4] L. Grafakos, A. Greenleaf, A. Iosevich and E. Palsson, Multilinear generalized Radon transforms and point configurations, arXiv:1204.4429, submitted(2012) 1, 3

[5] A. Greenleaf and A. Iosevich, On triangles determined by subsets of the Euclidean plane, the associated bilinear operators and applications to discrete geometry, Analysis and PDE, Vol 5, No. 2, 2012. 1, 6

[6] A. Greenleaf, A. Iosevich, M. Mourgoglou, On volumes determined by subsets of Euclidean space, arXiv:1110.6790, submitted (2011). 1

[7] V. Harangi, T. Keleti, G. Kiss, P. Maga, A. Mathe, P. Mattila and B. Strenner, How large dimension guaranteesa given angle?, arxiv:1101.1426v2, submitted (2012). 1

[8] A. Iosevich, M. Mourgoglou, E. Palsson, On angles determined by fractal subsets of the Euclidean space via Sobolev bounds for bilinear operators, arXiv:1110.6792, submitted (2011). 1

[9] J. Kahane, Sur la dimension des intersections, Aspects of Mathematics and its Applications, Vol. 34 (1986), 419-430. 6

[10] P. Mattila, Hausdorff dimension and capacities of intersections of sets in n-space, Acta Math. 152 (1984), 77-105. 6

[11] P. Mattila, Spherical averages of Fourier transforms of measures with finite energy: dimensions of intersections and distance sets, Mathematika, 34 (1987), 207-228. 1, 4, 6

[12] P. Mattila, Geometry of sets and measures in Euclidean spaces, Cambridge Univ. Pr., vol. 44 (1995). 6, 9

[13] C. Sogge, Fourier Integrals in Classical Analysis, Cambridge Univ. Pr., (1993) 4

[14] E. M. Stein, Harmonic Analysis, Princeton Univ. Pr. (1993). 9

[15] T. Wolff, Decay of circular means of Fourier transforms of measures, Int. Math. Res. Not. 10 (1999), 547-567.

Department of Mathematics, University of Rochester, Rochester, NY 14627

E-mail address: bliu19@z.rochester.edu 